\title{
Simulating the impacts of strong bus priority measures
}

\author{
B.J.Waterson ${ }^{\mathbf{a}}$, B.Rajbhandari ${ }^{\mathbf{b}}$ and N.B.Hounsell ${ }^{\mathbf{c}}$
}

Transportation Research Group, Department of Civil and Environmental Engineering, University of Southampton, Highfield, Southampton SO17 1BJ, UK.

\begin{abstract}
Policies to reduce levels of traffic congestion and pollution in major urban areas often focus strongly on the concept of a sustainable transport system, but to achieve this vision a significant modal shift from private car to public transport will be required. This paper reports on a recent research study which provides a framework within which to model the behavioral responses of travelers following the implementation of strong bus priority measures (where road capacity is deliberately removed from general traffic and given to buses). A summary of the different behavioral responses which can be expected is given and results from a practical implementation of the framework which has been based on two commercial transport modeling packages (CONTRAM and TRIPS) are discussed. These results suggest firstly that the effect of implementing such strong bus priority measures is as dependent on the characteristics of the local travelers as on the scheme itself and secondly that implementing too strong a scheme may not benefit public transport overall.
\end{abstract}

Keywords: Bus Priority, Traveler Behavior, Modal Change, Urban Congestion, Sustainable Transport

\section{Introduction}

Policies to reduce levels of traffic congestion and pollution in major urban areas often focus strongly on the concept of 'a sustainable transport system'. For example, in a recent U.K. Government White Paper on Transport (DETR 1998) it is stated that the bus will increasingly become the focus of an efficient transport system that gets people to where they want to be quickly and comfortably without having to rely on cars. Highly congested conditions have arisen because of continual growth in private car use both in North America and Western Europe. To achieve this vision of buses providing the core of a sustainable urban transport system (either within the ten year timescale of the U.K. White Paper plan or in the longer term), assuming that current levels of growth in the number of person trips being made is maintained, a significant reduction in the proportion of person trips made by car and an increase in the proportion made by bus will be required.

For this change in preferred mode of transport to occur, public transport must be perceived by urban travelers to be faster and more efficient than private cars. The reality at present however is that buses often suffer in the same congestion as cars and there is consequently no perceived advantage to travelers of choosing buses over private cars as their mode of travel.

Since urban traffic planners first identified the concept of bus priority, many different schemes have been implemented to try to protect buses from urban traffic congestion. These schemes have traditionally centered either on implementing bus only lanes to segregate the buses from the queues of general traffic, or providing priority to buses at signalized intersections (for example Hunter-Zaworski et al. 1995, Hounsell et al. 1996 and Mirchandani et al. 2001). Bus lanes however are often only implemented on a small section of the bus route and are usually designed such that throughput capacity for general traffic at intersections is hardly affected (e.g. through the use of set-backs for bus lanes (as in Figure 1) where the bus lane stops short of the junction allowing general traffic to utilize the full carriageway width). New facilities for bus priority at traffic signals can also provide worthwhile benefits for buses but again, implementation is usually within the context of maintaining capacity for general traffic at intersections. This requirement of scheme design, to maintain levels of general traffic flow, limits the benefits that can be achieved for public transport (as shown in Figure 1 where a bus is still delayed by other traffic). It has always been considered that if 'stronger' bus priority measures were implemented (those measures that intentionally restrict the roadspace available to private cars in order to create maximum benefit for buses) then the combined carrot and stick effect would become much more pronounced in favor of public transport and greater levels of modal change could then be achieved. 


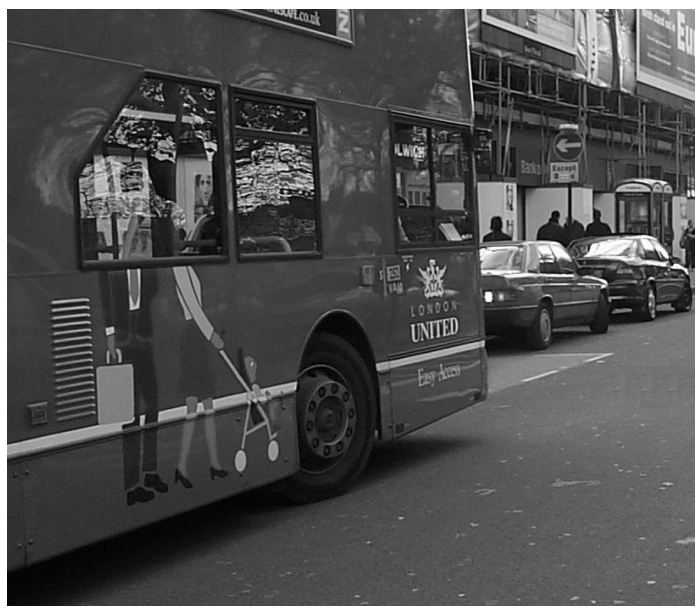

Figure 1 : A bus lane set-back causing buses to be delayed by general traffic.

This paper reports on the simulation strand of a recent research project (sponsored by the British government research organization EPSRC - Engineering and Physical Sciences Research Council) carried out within the Transportation Research Group of the University of Southampton, U.K.. Although research has been carried out on the impact of bus priority measures on other traffic on the same road (Shalaby 1999), there is evidence to suggest (Cairns et al. 1998 and MVA 1998) that the effects on the wider network must be considered when assessing the impact of such schemes. The project therefore set out to provide a framework to model the implementation of strong bus priority measures in an urban network and the responses of travelers faced with the increased levels of congestion for general traffic that the priority measures generate. In this way it was hoped to be able to assess whether such measures really could lead to the desired levels of modal change.

The study began by looking for examples of strong bus priority schemes that had already been introduced. It was found however that, although many schemes had been suggested, only those that had been significantly 'weakened' (often by alterations to reduce the adverse effect on general traffic) had ever been implemented. Priority schemes had also been implemented as part of a wider package of measures such as revised fare structures or timetables, arrival time displays at boarding points or introductions of complete new routes. This made it very difficult to isolate the effects of the priority scheme from the effects of the accompanying measures. The main reason given for the reluctance to implement strong bus priority measures was a lack of understanding as to precisely what the short term and long term effects of such schemes would be. The framework therefore is designed to provide planners with the information they need to make informed decisions on the viability and consequences of strong bus priority measures.

\section{Traveler Behavioral Responses}

It is widely accepted that following a disruption to their normal journey, such as encountering the increased levels of congestion caused by a road accident, car drivers will change their journey by the minimum amount necessary (for example taking the minimum diversionary route). This situation also occurs in the case of longer term disruption such as road works or the implementation of a strong bus priority measure, but the longer timescale in these cases means that the behavioral responses can be more complex, wide-ranging, and take more time to be revealed (Cairns et al. 1998). In summary, drivers can be expected to respond in one or more of the following ways:

\section{Do nothing.}

Despite the advanced publicity that would need to accompany the introduction of for example a strong bus priority scheme, many drivers initial response will be to continue trying exactly the same journey as before. The evidence to support the existence of this as the initial behavioral response comes from studies that have noted severe congestion in the first few days following implementation of bus priority schemes (Cairns et al. 1998).

\section{Re-routing.}

When car drivers become aware that their initial "do nothing" response is not sufficient, their next response is expected to be a change to an alternative route. This has been observed to create a "ripple effect" (MVA 1998) in congestion in both time and space (see Figure 2), where traffic diverting to routes parallel to that where the 
bus priority measure has been implemented cause increased congestion there, which causes some vehicles from these routes to divert outwards again and so on.

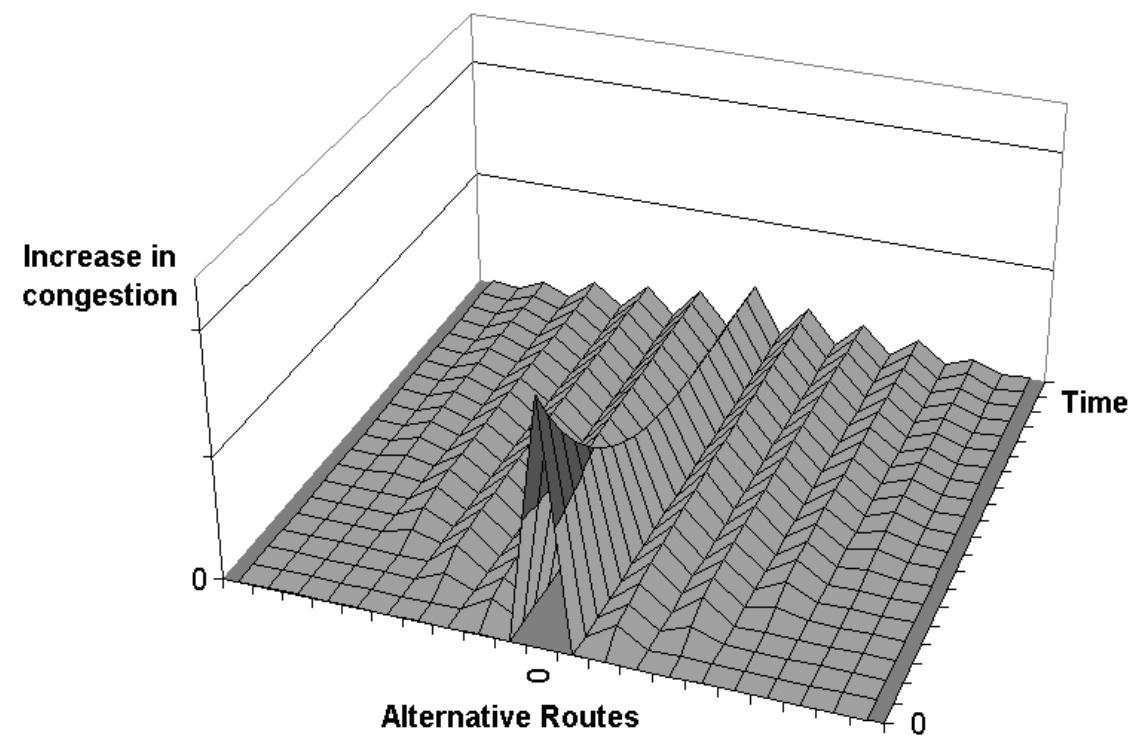

Figure 2 : The ripple effect of diverting traffic onto alternative routes.

3. Re-timing.

If changing route does not sufficiently alleviate the congestion being experienced, drivers may consider altering their time of departure so that their journey takes place either before the congestion is at its highest or after the queues have dissipated. This effect, commonly referred to as peak-spreading, has been studied by, amongst others, Al-Azzawi 1997, van Vuren et al. 1999 and Ivan and Allaire 2001.

\section{Modal change.}

It is this response that is the aim when strong bus priority measures are implemented, as it represents a change away from private car use (and in the longer term car ownership) and towards more sustainable forms of transport (buses in this case). Other responses that can also occur at this point in the decision process include an increase in car-sharing or multi-mode journeys where a traveler will (for example) drive to a point outside the congested area and then transfer to a bus for the remainder of their journey.

\section{Trip suppression.}

The final response of travelers when all other options have been exhausted is not to make the trip. This may be represented by a change in destination (for example using a different shopping mall if the congestion around one is considered just too high), or a reduction in the number of trips (one large shopping trip instead of two smaller ones). In the longer term these decisions are likely to have an influence on land use patterns. Within the framework, travelers were modeled to take this decision if their perceived travel cost $(\$ / \mathrm{km})$ exceeded predefined thresholds for both the journey by private car and the journey by public transport. Evidence from a wide range of schemes that appears to support the theory that capacity reduction for general traffic results in trip-suppression in the same way that increased capacity (such as road building) results in trip-generation is summarized in Cairns et al. 1998.

In reality these responses are often not independent of each other. As an example, because the same journey by bus or car may take different durations, the departure time may need to change at the same time as the mode in order to maintain the same arrival time (most likely to occur for example in the morning commute when the traveler needs to be at the office at a certain time). The stronger the priority scheme being proposed, the more of these responses that travelers will be forced to consider and therefore it is necessary for modeling purposes to consider each in turn, as in Figure 3. To realistically model behavior however, the framework must allow all previous responses to be reconsidered at each stage of the process. 


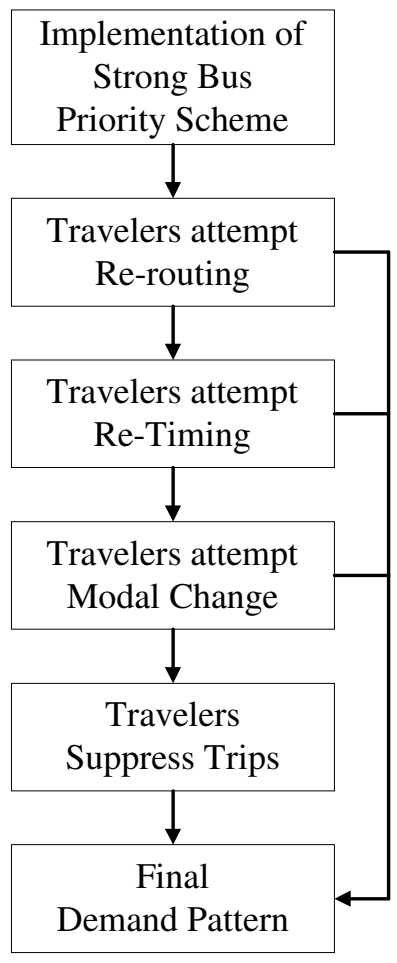

Figure 3 : Expected order of behavioral responses.

\section{Modeling Framework}

Traditional traffic simulation models (whether microscopic vehicle interaction models or macroscopic assignment models) take as their base unit an individual vehicle or group of vehicles. Because of the need in this study to consider the individual characteristics of each traveler (for example whether the trip being made is a work or non-work trip changes the perceived value of time), in order to accurately assess the affects on traffic flows of the potential behavioral responses it was necessary to define the modeling framework to have as a base unit an individual traveler rather than a vehicle.

Ideally, a practical implementation of the framework could be based around a single, well-established, validated urban traffic simulation model. Unfortunately, no single model was identified that incorporates the appropriate congestion dynamics and public transport functions. A practical implementation of the framework was therefore achieved by combining two commercially available software packages CONTRAM and TRIPS (for which calibrated test networks were already available) into a nested iterative model as illustrated in Figure 4.

CONTRAM (White et al. 1994) is a macroscopic urban traffic model which when given a network topology and vehicle demand matrix, assigns the vehicles to the network to minimize a generalized cost objective function. This is done by the model calculating the queue or delay and therefore the travel time on each 'link' of the network (unidirectional section of road between two intersections) in each time period and these are then used to determine the optimum route between each origin-destination pair. The total travel time, distance and tolls encountered on this optimum route are then output. CONTRAM results can therefore be used to assess, in conjunction with the corresponding driver characteristics, the perceived costs of traveling by private car using an equation such as (1) where the precise value of each of the coefficients would need to be determined for the travelers being affected by any proposed scheme. The value of $\mathrm{V}_{\mathrm{t}}$ will depend on the trip purpose and $\mathrm{P}$ must account for time spent searching for a parking space and walking to the traveler's final destination as well as any monetary cost.

$$
\mathrm{PC}_{\mathrm{C}}=\mathrm{V}_{\mathrm{t}} * \mathrm{IV}_{\mathrm{t}}+\mathrm{VOC}_{\mathrm{km}} * \mathrm{D}+\mathrm{T}+\mathrm{P}
$$

It should be noted here that the inclusion of road tolls and perceived parking charges in the cost function potentially enables this framework also to be used to assess the behavioral responses to transport system management policies such as 'auto restricted zones' 'parking supply reduction' (Khisty 1990). 


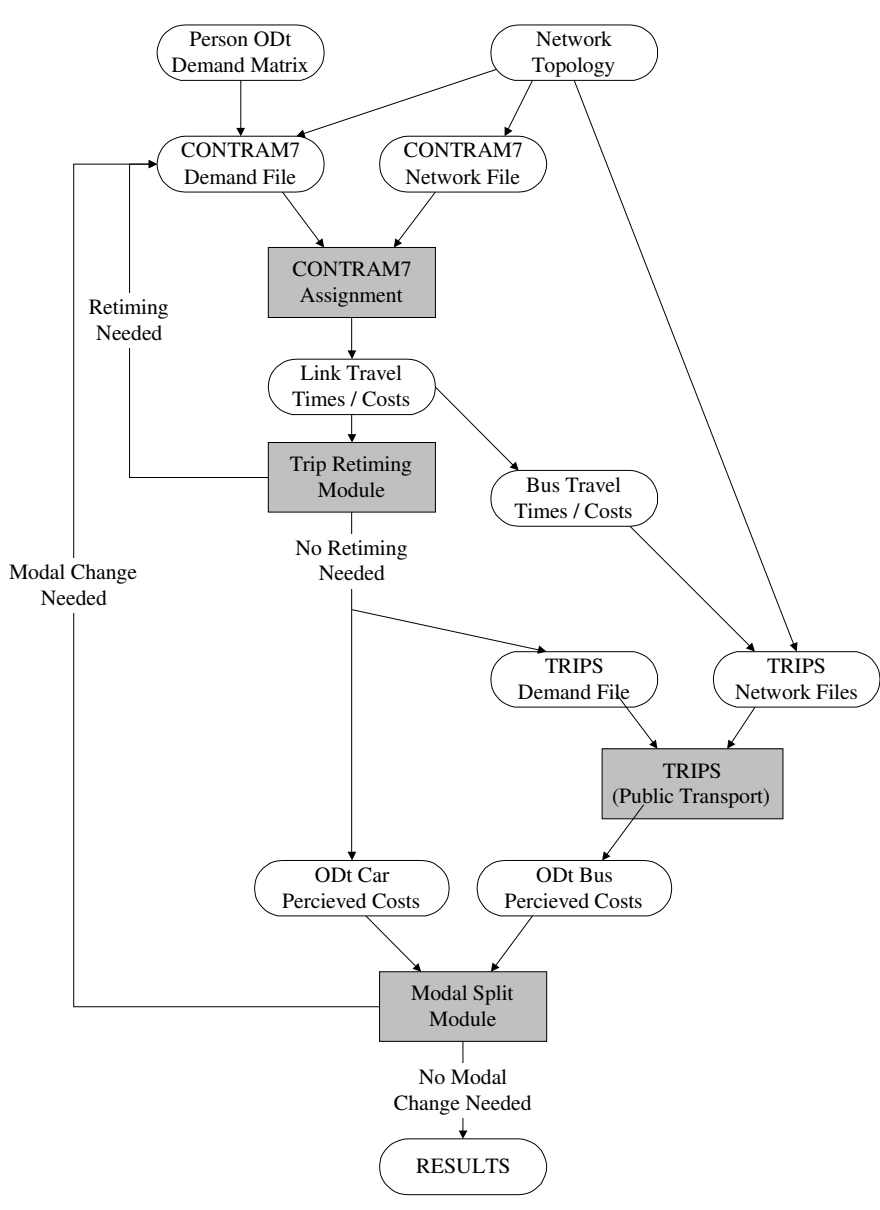

Figure 4 : The nested iterative model.

Unfortunately, although the CONTRAM assignment includes buses (and any other classes of vehicle specified by the user), because it has vehicles rather than travelers as its base unit, there is no way to realistically calculate the perceived costs of traveling by public transport from CONTRAM outputs alone. To illustrate the problem consider the following situation:

A person wishes to travel by bus for the whole length of a bus route. Because CONTRAM has a vehicle traveling between the start of the bus route (origin) and end of the bus route (destination) it outputs the optimum time and distance for this origin-destination pair (traveling along the prescribed route). Now suppose however that the person instead wishes to alight at a stop halfway along the route. Because the vehicle does not stop here, only the traveler, CONTRAM does not provide outputs for this journey.

To summarize the problem, because vehicles in CONTRAM are defined only by their origin and destination, there is no concept of a person being able to board or alight from a bus at an intermediate point on its journey. The issue is further complicated if the origin and destination of the traveler do not fall on the same bus route, leading to the traveler needing to change bus en-route.

The public transport model of the TRIPS traffic simulation suite (TRIPS 1995) however is ideally suited to assessing the time, distance and fares incurred for travelers using public transport. It is able to model all phases of a journey by public transport, including walking to the initial boarding point, waiting at that point for the arrival of a bus, in-vehicle travel time, changes to secondary bus routes and walking from final alighting point to final destination. Unfortunately, the model is not designed to represent the time varying congestion conditions experienced during for example the morning commute. Taking travel times from the CONTRAM output however and applying the TRIPS model to a series of short 'time-slices' corresponding to those used in CONTRAM, enables these time variant factors to be incorporated and the perceived cost by traveling by public transport can then be assessed by an equation such as (2). The higher weighting of $\mathrm{OV}_{\mathrm{t}}$ is required as research has shown the perception of this to be higher than that of $\mathrm{IV}_{\mathrm{t}}$.

$$
\mathrm{PC}_{\mathrm{B}}=\mathrm{V}_{\mathrm{t}} *\left(\mathrm{IV}_{\mathrm{t}}+2 * \mathrm{OV}_{\mathrm{t}}\right)+\mathrm{F}
$$


With the link between the outputs of CONTRAM and the inputs to TRIPS, the perceived costs of traveling by private car and public transport at different departure times for each traveler in the framework can be estimated and compared and any necessary trip-retiming, modal change or trip-suppression responses assessed accordingly. As noted above, if the congestion effects of implementing the strong bus priority measure can be negated by (for example) re-routing alone, then it is unnecessary for the trip-retiming, modal change and tripsuppression stages of the nested iterative model in Figure 4 to be carried out. When all traveler decisions have been assessed, the final 'cost' of the strong bus priority scheme can be estimated by calculating the actual resource cost for each traveler $\left(\mathrm{RC}_{\mathrm{C}}\right.$ or $\left.\mathrm{RC}_{\mathrm{B}}\right)$ rather than their perceived cost $\left(\mathrm{PC}_{\mathrm{C}}\right.$ or $\left.\mathrm{PC}_{\mathrm{B}}\right)$.

The same equations cannot be used to calculate the resource costs of travelers who decide not to make their trip. While the distance traveled, journey time, fares and tolls (and hence the resource cost) that these travelers would have faced if they had traveled on their optimum route can be calculated, this is not the resource cost that is actually incurred by society. Instead, the true resource cost can be calculated using the economics concept of indifference and the thresholds used to make the trip suppression decision as follows. Within the framework, travelers were modeled to take this decision if their perceived travel cost $(\$ / \mathrm{km})$ exceeded predefined thresholds for both the journey by private car and the journey by public transport. However, at the point where their travel cost equals (the greater of) these thresholds they would be cost indifferent to whether they travel or not. This value, the threshold multiplied by the distance that would be traveled must therefore be their cost of not traveling and is thus the resource cost used in the results.

\section{Test Scenarios}

As a demonstration of the framework in operation, a series of theoretical strong bus priority schemes were designed on a small sub-network of the city of Southampton, U.K. (see Figure 5). The 'key-hole' shaped subnetwork was chosen because it incorporates the central business district at the southern end, with three major arterials (and links between them) to provide drivers with alternative routing options, but is bounded on the East side by a river and on most of the West side by a large area of parkland. This bounding is important because it prevents the need to consider results for the whole city network, which may lead to the effects of the strong bus priority scheme being lost in general traffic variability. Coefficients for equations (1) and (2) were derived from standard values (DMRB 1997), local transit operators and existing research on the network (Waterson et al. 2001) as appropriate.

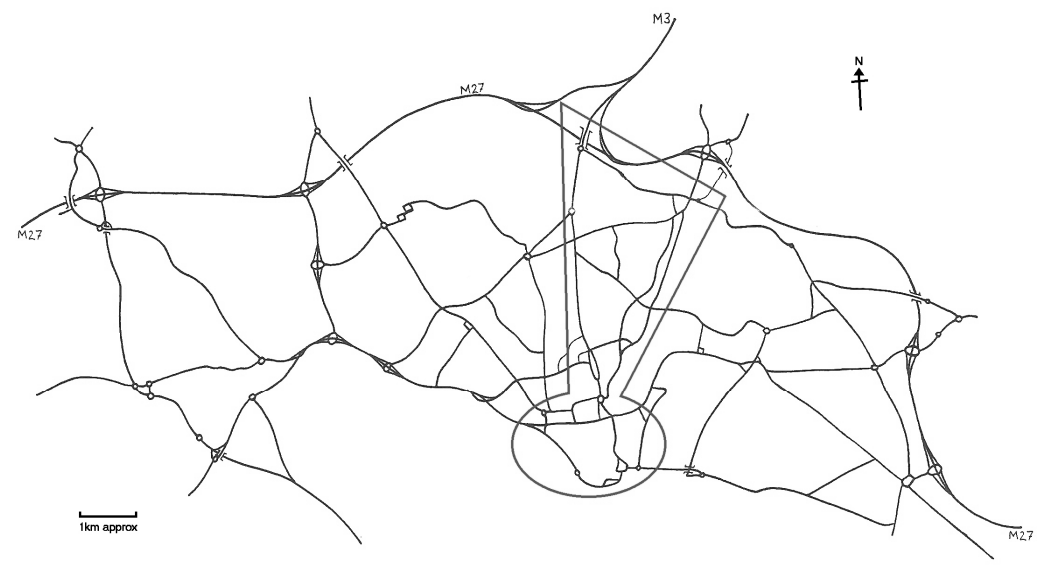

Figure 5 : Map of Southampton showing test sub-network.

\section{Base Network Results}

The first important results to come out of the framework demonstration were not concerned with strong bus priority measures. Instead these results relate to the relative perceived costs of traveling by public and private transport in the base network (i.e. the network before any priority schemes have been implemented). Figure 6 shows the (circa) 5500 travelers whose journey is completely contained within the test sub-network. From this graph it is clear that the (standard) assumptions of a certain proportion of travelers having to pay for city-center parking and a certain proportion perceiving themselves to be in working time (approximately $15 \%$ for car drivers, DMRB 1997) with the correspondingly higher perceived value of time, lead to travelers falling into four almost distinct groups (labeled A,B,C and D). 


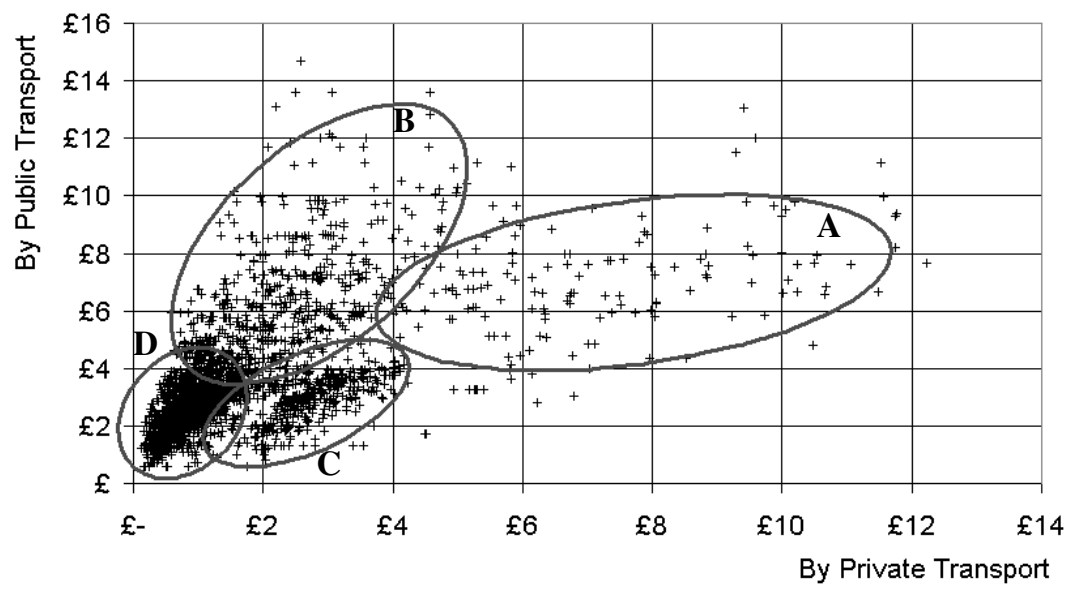

A Working + Pay

B Working + PNR

C Non-Working + Pay

D Non-Working + PNR

Figure 6 : Perceived Cost of Travel Groups.

The relative positioning of these groups is determined horizontally by the perceived availability and cost of parking and vertically by the perceived availability of public transport. By definition a strong bus priority measure should reduce the perceived cost of traveling by public transport and increase that by private car. This can be represented graphically (as in Figures $7 \mathrm{a}$ and $7 \mathrm{~b}$ ) by a move in the equality line where the two perceived modal travel costs are equal, with the potential modal change that can be achieved being approximately the number of travelers who fall between the two lines. Figure 7a illustrates a case where some travelers in all four groups perceive their cost of traveling by public transport to be only slightly higher than that of traveling by private transport, a small change therefore could produce a significant modal change. Figure $7 \mathrm{~b}$ however illustrates a case where all travelers face significant differences between their perceived costs of travel and hence the potential for causing modal change is much more limited.

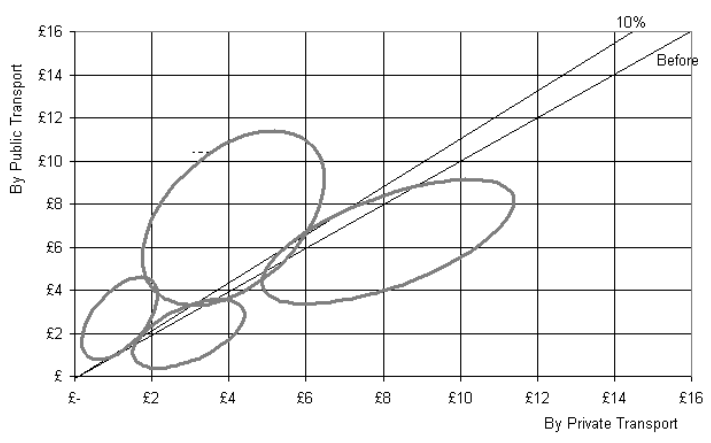

Figure 7a : Potential Modal Change.

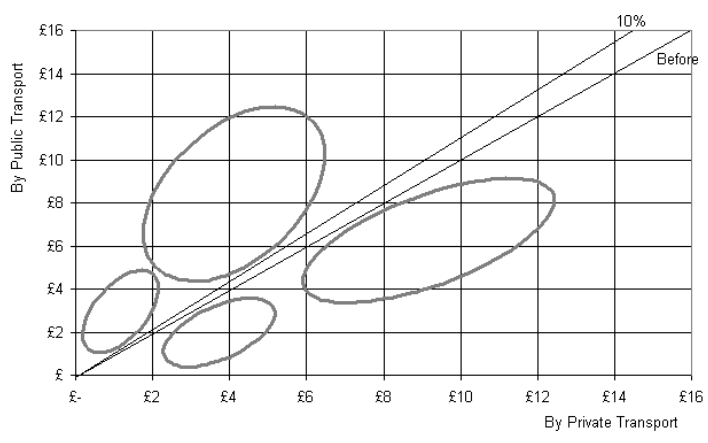

Figure $7 \mathrm{~b}$ : No Potential Modal Change.

This graphical method is only an approximation, both to the results obtained using the framework and reality however, because the priority measure will not affect all travelers in the network equally and some of the increase in private transport costs will be negated by re-routing or re-timing decisions. Even given this limitation, it is clear from Figures $7 \mathrm{a}$ and $7 \mathrm{~b}$ that the potential modal change is as much dependent on the location of the four groups as on the size of the effect of the strong bus priority measure (which in these figures has resulted in a $10 \%$ shift in relative perceived travel costs).

\section{Priority Scheme Results}

The second important result to come from the test scenarios was the effect of the length of the strong bus priority scheme on the benefit that it produced for public transport. Different lengths of schemes were tested on the "Portswood Road" arterial, a $48 \mathrm{~km} /$ hour single mixed lane of traffic in each direction in the base network, with peak bus flows in the region of 25 per hour on some sections. The schemes involved restricting traffic to buses only southbound (towards the central business district) and varied in length between a closely targeted $300 \mathrm{~m}$ stretch in the most congested section of the route and a $3 \mathrm{~km}$ long measure effectively encompassing almost the entire arterial. 
As the length of the priority scheme was initially increased, the benefit to public transport (reduction in perceived travel cost) initially increased and the dis-benefit to private transport also increased (up to $8 \%$ averaged across all travelers in the sub-network). As the length of the measures was increased further however up to the $3 \mathrm{~km}$ length, although the perceived cost of traveling by private transport continued to rise, the time benefits to public transport actually began to decrease. A detailed examination of vehicle travel times with this very strong measure implemented suggested that the congestion in the network at each end of the scheme was now so severe that the delay to buses (compared to travel times in the same sections of the network before the strong bus priority scheme was implemented) was now beginning to outweigh the benefits gained within the boundaries of the scheme itself.

\section{Conclusions}

This research study has attempted to provide a framework to enable local traffic authorities who are considering implementing strong bus priority measures to accurately assess their potential impacts on the urban network. To be successful the framework had to allow for a wide range of possible behavioral responses of travelers, many of which are themselves the subject of on-going research. A practical implementation of the framework has been described, based around two commercially available transport simulation models (CONTRAM and TRIPS) and the framework has been tested on a small sub-network of the city of Southampton, U.K.. Initial results have shown that precise knowledge of the local traveler population is essential as this can be as important to the potential impacts of the schemes as the priority measures themselves. Results have also indicated that it is possible to implement too strong a scheme of bus priority measures, and that doing so can actually result in an overall dis-benefit to public transport services. This result would not have been available to transport planners without the framework developed in this project.

\section{Acknowledgements}

The authors wish to thank the U.K. Engineering and Physical Sciences Research Council (EPSRC) for their support of this research and for the many local authorities across the U.K. who provided help, expertise and data. 


\section{Appendix I. References}

- A-Azzawi, M. (1997). "An overview of three techniques designed to aid planners with over-assignment and peak spreading in traffic modelling studies." Traffic Engineering and Control, 38(11), 604-606.

- Cairns, S., Hass-Klau, C., and Goodwin, P. (1988). Traffic impact of highway capacity reductions: Assessment of the evidence. Landor, London.

- Department of the Environment, Transport and the Regions (DETR) (1998). A new deal for transport: Better for everyone. The Stationery Office, London.

- Design Manual for Roads and Bridges (DMRB) (1997). Highways Economics Note No.2. Volume 13, Section 2, The Stationary Office, London.

- Hounsell, N. B., McLeod, F. N., Bretherton, R. D., and Bowen, G.T. (1996). "PROMPT: Field trial and simulation results of bus priority in SCOOT." Proc. 8th Int. Road Traffic Monitoring and Control Conf., IEE, London, 90-94

- Hunter-Zaworski, K. M., Kloos, W. C., and Danaher, A. R. (1995). "Bus priority at traffic signals in Portland: The Powell Boulevard pilot project.” Transp. Res. Record 1503, 29-33.

- Ivan, J. N., and Allaire, S. A. (2001). "Regional and area-type modeling of peak spreading on Connecticut freeways." J. Transp. Engrg., 127(3), 223-229.

- $\quad$ Khisty, C. J. (1990). Transportation engineering. Prentice-Hall, N.J..

- Mirchandani, P., Head, L., Knyazyan, A., and Wu, W. (2001). "An approach towards the integration of bus priority and traffic adaptive signal control." Proc. 80th TRB Conf. TRB, Washington D.C..

- MVA. (1998). Traffic Impact of highway capacity reductions: Report on modelling. Landor, London.

- Shalaby, A. S. (1999). "Simulating performance impacts of bus lanes and supporting measures." J. Transp. Engrg., 125(5), 390-397.

- $\quad$ TRIPS An introduction to public transport modelling - version 7. (1995). MVA Systematica, Woking.

- $\quad$ van Vuren, T., Carmichael, S., Polak, J., Hyman, G., and Cross, S. (1999). "Modelling peak spreading in continuous time." Proc. Seminar F, European Transport Conf., PTRC, London, 93-105.

- Waterson, B.J., Hounsell, N.B., and Chatterjee, K. (2001). "Quantifying the potential savings in travel time resulting from parking guidance systems - a simulation case study" JORS 52(10), 1067-1077.

- White, C., Taylor, N., and Hounsell, N. (1994). "CONTRAM - a computer suite for modelling road congestion." Traffic Technology International '94, T. Robinson, ed., UK and International Press, Dorking, 106-110.

\section{Appendix II. Notation}

D Distance traveled $(\mathrm{km})$.

F $\quad$ Fares incurred during a journey (\$).

$\mathrm{IV}_{\mathrm{t}} \quad$ Travel time spent in-vehicle (hours).

$\mathrm{OD}_{\mathrm{t}} \quad$ Origin-destination-departure time specific.

$\mathrm{OV}_{\mathrm{t}} \quad$ Travel time spent out of vehicles (hours).

$\mathrm{P} \quad$ Parking cost (\$): Including searching and walk time.

$\mathrm{PC}_{\mathrm{B}} \quad$ Perceived cost of traveling by public transport $(\$)$.

$\mathrm{PC}_{\mathrm{C}} \quad$ Perceived cost of traveling by private car $(\$)$.

PNR Private Non-residential Reserved parking space.

$\mathrm{RC}_{\mathrm{B}} \quad$ Resource cost of traveling by public transport (\$).

$\mathrm{RC}_{\mathrm{C}} \quad$ Resource cost of traveling by private car $(\$)$.

$\mathrm{V}_{\mathrm{t}} \quad$ Value of time (\$/hour) : Dependent on trip purpose.

$\mathrm{VOC}_{\mathrm{km}} \quad$ Vehicle operating costs $(\$ / \mathrm{km})$.

$\mathrm{T} \quad$ Tolls incurred during a journey $(\$)$. 